\title{
Research Paper: Small Ball Exercise Program for Patients With Chronic Nonspecific Low Back Pain: A Randomized Clinical Trial
}

\author{
Ghazaleh Vahedi ${ }^{1}$, Zahra Mosallanezhad ${ }^{2,3^{*}}$, Yahya Sokhangooi ${ }^{3}$, Hamideh Abyaneh Miri' $^{2}$, Fakhrosadat Jafari Mousavi ${ }^{2}$, Maryam Ghodrati ${ }^{2}$, \\ Fatemeh Ehsani ${ }^{4}$, Afsun Nodehi Moghadam ${ }^{2,3}$ \\ 1. Department of Sport Biomechanics, Faculty of Physical Education \& Sport Sciences, Central Tehran Branch, Islamic Azad University, Tehran, Iran \\ 2. Department of Physiotherapy, University of Social Welfare and Rehabilitation Sciences, Tehran, Iran. \\ 3. Research Center on Aging, University of Social Welfare and Rehabilitation Sciences, Tehran, Iran. \\ 4. Rehabilitation Research Center, Department of Physiotherapy, School of Rehabilitation, Semnan University of Medical Sciences and Health Services, Semnan, Iran
}

Citation: Vahedi Gh, Mosallanezhad Z, Sokhangooi Y, Abyaneh Miri H, Jafari Mousavi F, Ghodrati M, et al. Small Ball Exercise Program for Patients With Chronic Nonspecific Low Back Pain: A Randomized Clinical Trial. Physical Treatments. 2016; 6(2):71-78. https://doi.org/10.18869/ nrip.ptj.6.2.71

: https://doi.org/10.18869/nrip.ptj.6.2.71

Article info:

Received: 28 Dec. 2015

Accepted: 01 May 2016
Keywords:

Back pain, Muscle imbalance, Physical therapy, Exercise therapy

\begin{abstract}
A B S T RA C T
Purpose: Low back pain (LBP) is one of the main causes of disability in adults. Approximately $80 \%$ of LBPs lead to chronic nonspecific low back pain (CNSLBP). Damages to back region may be due to weak musculoskeletal structure, impaired muscle or joint flexibility, changes in muscle tone, and reduced strength and endurance. A set of exercises by small ball was designed by the corresponding and first authors to improve the balance of muscular activity. The current study aimed at evaluating the efficacy of small ball exercise program compared to Williams exercises, in patients with nonspecific chronic low back pain.

Methods: Among the patients referred to the physical therapy clinics in Tehran, Iran, 30 cases (16 males and 14 females) were selected, considering the inclusion and exclusion criteria, through accessible sampling method in 2014-2015. Demographic information was recorded. The patients were randomly allocated into one of the intervention groups. For both intervention groups, a 10-session routine electrotherapy was applied (transcutaneous electrical nerve stimulation, hot pack, and ultrasound). Additionally, in the intervention group 1, patients received small ball exercises, but the intervention group 2 received Williams exercises (as the control). Treatment was conducted by a physiotherapist and a research fellow assessed pain intensity based on visual analogue scale (VAS) and disability index (Oswestry). Assessments were performed before starting the intervention, after treatment, and then after 2 weeks of follow-up. Paired and independent samples $t$ tests were employed to conduct the statistical analysis by SPSS18.
\end{abstract}

Results: There was no significant difference between the groups based on the baseline characteristics. In both groups, pain and disability showed significant improvement $(\mathrm{P}<0.05)$. But in small ball exercise group, reduction in pain $(\mathrm{P}=0.004)$ and disability $(\mathrm{P}=0.03)$ were more noticeable even after 2 weeks of follow-up. The applied treatment program was reported more acceptable and effective by patients.

Conclusion: The current study, while proving beneficial effects of Williams exercise with routine physiotherapy in patients with CNSLBP, showed that substitution of these exercises by small balls exercises could enhance the effect of treatment on pain and disability. Small ball exercise program also showed better outcome in the follow-up, and was ranked higher regarding its impact, ease of implementation, and desirability by the patients.

\footnotetext{
* Corresponding Author:

Zahra Mosallanezhad, PhD

Address: Department of Physiotherapy, University of Social Welfare and Rehabilitation Sciences, Tehran, Iran.

Phone: +98 (21) 22180039

E-mail: zmosallanezhad@yahoo.com
} 


\section{Introduction}

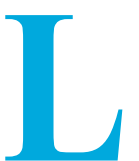

ow back pain (LBP) is one of the most common health problems worldwide [1]. There are different treatment methods, but studies could not present the most effective treatment, which may be due to the complexity of factors contributing to $\operatorname{LBP}[2,3]$. Various theories have been developed based on different views on the causes of back pain $[3,4]$. Poor neuromuscular body control is proposed as a possible factor [5]. In general, one of the major causes of low back pain is pathobiomechanical factor, which often leads to imbalance in the muscles around the spine $[5,6]$. Coordinated musculoskeletal elements of lower back, thoracic, and neck regions provide the stability of the spine, and also transmit the forces applied to the body to lower extremities in static and dynamic positions [7]. Various reasons, such as muscle weakness and tightness of some other groups of muscles, disturb the protective role of muscles and force transfer in this part $[7,8]$. It leads to a change of torque forces exerted on the spine or in many cases disrupts the corresponding muscle activity timing, which declines the capacity of musculoskeletal system to withstand and transfer the forces, and subsequently causes deformity, pain, and disability [9].

The role of motor system is emphasized in causing and managing low back pain [10]. Motor system balance is the result of good neuromuscular coordination and a well-adjusted system of opposing muscle activities. It is very important in balanced and precise movement [710]. The repetitive movements and long-term positions can change the responses of neuromuscular system that in turn can lead to impaired muscle function and change the pattern of motion, and finally appear as pain or movement impairment [11]. Muscle imbalance means the changes in the pattern of agonist and antagonist muscle activities during function. Hence to reduce pain, movement impairment should be treated and altered muscle activation patterns should be corrected $[7,12]$.

Some other studies emphasize on the activation and modification of the global and mover muscles activities around the lower back and pelvic region [13]. Imbalance in the muscles of lower back and pelvic region leads to dysfunction in these areas and lower extremities. Correction of the function and activity of these muscles can resolve abnormalities and decrease the symptoms and complaints of the patients $[7,9,12,13]$.

Prescription of appropriate exercises, which activate muscle groups, would help relieve muscle imbalance. The degree of acceptability and success of an exercise program depends on its level of difficulty, attraction, and practicality for patients. Performing an appropriate exercise program can play an important role in reducing symptoms and complaints in patients [13]. Therefore, the current study aimed at investigating the effect of a new exercise program designed by authors on declining pain and disability of patients with chronic nonspecific low back pain (CNSLBP), in comparison to the Williams exercise programs routinely used in physiotherapy clinics.

A set of exercises were designed using a small ball to use the activity of muscles connecting to lower back and pelvis. At the presence of muscle imbalance, postural muscles get tight and phasic muscles get weak. To manage muscle imbalance, it is tried to relax tight muscles and strengthen weak ones. Based on the intensity and type of muscle contraction, both inhibitory and stimulatory effects on muscles can be obtained. Through the mechanisms of post isometric relaxation and reciprocal inhibition, a period of relaxation occurs after the muscular effort. Muscle strengthening is also expected through recruiting muscle activation force against the resistance provided by the ball.

\section{Materials and Methods}

\section{Study design and participants}

The current Randomized Clinical Trial (RCT) was conducted in 2014-2015, Tehran, Iran. Volunteers with CNSLBP were briefed about the study and signed consent forms to participate in the study. On the basis of study inclusion/exclusion criteria, 30 participants were selected and randomly allocated into groups of 1 and 2 . Group 1 received routine electrical therapy plus exercise program with small ball, and group 2 received routine physical therapy plus Williams exercise program. The application of interventions was randomly assigned to each group. Treatment in each session was performed by a physiotherapist, and the assessment of patients in both groups was performed by a research fellow independently. Assessor was trained to assess methods and prevent some important biases.

The physiotherapist, who was responsible for the treatment, was blinded to the random allocation and assessment, and the participants and the research fellow were also blinded to the random allocation and the kind of exercise program. Demographic data, including age, gender, height, weight, and job were collected through questionnaires. The improvement of patients was assessed by evaluating the severity of pain and disability. Measurements were performed by a research fellow with the relevant instruments. Assessments were carried out at 
the beginning of the treatment (before the intervention), at the end of each session, and finally 2 weeks after completion of therapy sessions in both groups, as a follow-up. Pain intensity was measured by Visual Analogue Scale (VAS); the patients rated their lower back pain on a scale from 0 (the lowest pain) to 10 (the highest pain) [14].

Disability related to back pain was assessed using the Oswestry low back pain disability questionnaire (the questionnaire is attached) $[15,16]$. The inclusion criteria were no special cause for low back pain, worsening of the pain with more activity and relieving by rest, experiencing at least 6 weeks of low back pain with different intensities at least twice a year, each time at least for 1 week. The exclusion criteria were history of fractures in the spine and limbs, damaged intervertebral disk, vertebral joints, and pelvic dysfunctions, history of surgery, tumor development, infection, radiculopathy, rheumatoid arthritis, anatomical abnormalities, dizziness, untreated visual impairment, metabolic and neurological disorders, arthrosis, drug addiction, and using relaxation and sleeping medication or alcohol at least 1 week before the test. The study was approved by the Ethics Committee of the Islamic Azad University, Central Tehran branch.

\section{Intervention protocol}

For both groups, a set of electrotherapy was applied at the same points of lumbar and gluteal regions, for 10 sessions, every other day. Electrical treatment was the same for both groups, including transcutaneous electrical nerve stimulation (TENS) for 20 minutes, ultrasound for 4 minutes, hot pack, infrared (IR), massage, and cupping $[17,18]$. Exercise protocol was given to 2 groups differently; group 1 was taught the small ball exercise, and group 2 Williams exercise [20]. Both groups performed their exercise programs 3 times a day, started from a set of 6 for each exercise, holding 6 seconds, and gradually increased to a set of 25 for each exercise.

\section{Group 1: Small ball exercises}

1) In crook lying position with knee bent, the ball is put between the knees and let the thigh adductors contract and push the small ball. Commonly, adductors predispose to tightness and shortness. This exercise through post isometric relaxation mechanism makes adductors relaxed.

2) In crook lying position with knee bent, put the ball out the knees and push the ball with knees one by one, activating the Tensor Fasciae Latae (TFL) and gluteus medius muscle.
3) In crook lying position with knee bent, put the ball out the knees and push the ball with both knees to activate the multifidus muscles of contralateral side, and push the ball with knees one by one to activate the TFL and gluteus medius muscles.

4) In supine position with knee and hip at $90^{\circ}$ flexion, put the ball on the palm of one foot and push the ball toward the wall. On the other side, bring one knee to the abdomen and the resistance created by hand prevents to do so. In this exercise, once the pelvis is opened once from up and once from down, this will cause balance of the muscles that are attached to the pelvis from top and bottom.

5) In the next exercise, the patient lies in supine with buttocks on the floor and knees bent, puts the ball in the sole of the feet and pushes the ball with both feet. In this case, the entire back muscles including the erector spinae and gluteus maximus are activated.

6) In the side lying position, the patient puts the ball on the lower back and gradually moves upwards to catch all segments; the patient is recommended to push the ball in each segment, pull-in his/her abdomen, and press the lower back to the ball. In this exercise, the contraction of the abdominal muscles and also the erector spinae are expected.

7) In the next exercise, the patient is in side lying position and puts the ball in the sole of the upper foot and pushes; in this exercise the quadratus lumborum muscle is activated. One of the main causes of low back pain is quadratus lumborum tightness. This muscle creates hip hike; this exercise acts exactly the opposite of mechanism that created it. Quadratus lumborum pulls the hip up. In this exercise it is opposite; with pushing the ball, the foot is pressed down. This causes muscle relaxation through the reciprocal inhibition mechanism.

8) The patient is in side lying position and sleeps toward the wall, puts the ball on the anterior superior iliac spine, in front of the thigh, and pushes the ball. In this exercise, isometric contraction of rectus femoris is taken.

\section{Group 2: The Williams exercises}

1) In supine position, knees are bent, then, the abdominal muscles are contracted and it is tried to bring lower back to the surface and hold it for 10 seconds.

2) In supine position, one knee is bent toward the chest and held with hands and at the same time the abdominal muscles are contracted and it is tried to bring 
lower back to the surface, while the other foot is kept flat on the ground.

3) In supine position, hands form a ring and take both knees toward the chest with the help of hands, then, legs are brought down and the feet are put on the ground.

4) In supine position, knees are bent and the feet are put on the ground, then, both knees are turned to the right and left.

5) In supine position, knees are bent, then, the head and shoulders are lift off the ground and held it for 10 seconds.

6) In supine position, knees are bent, and feet are put on the ground. Hands form a ring and take both knees toward the chest and the head and shoulders lift off the ground and it is tried to put hands on knees and hold for 10 seconds.

7) In supine position, one knee is brought into the abdomen and the opposite hand is put on thigh and resistance is created against the movement of the knee; it is held for 10 seconds.

8) In supine position, knees are bent, then the abdominal muscles are contracted, the patient should slowly go backwards until the hips are out of bed and the lower back is stuck to the ground, then, the hips are slowly brought forward until low back takes distance from ground.

9) In sitting position, knees are bent, the patient should slowly go back, put head and shoulders close to the ground, hold it for 10 seconds and slowly get back to the previous position.
Answering relevant questions, the participants also rated their exercise program in terms of its effectiveness, ease of implementation, and desirability.

Statistical analysis was performed using SPSS, version 18. Paired t test was used to evaluate the difference of indexes before, between, and after treatment in each group, and independent $t$ test was used to compare the differences between the 2 groups regarding the baseline characteristics.

\section{Results}

Overall, 16 males and 14 females participated in the study. The results showed that in both groups, distribution of variables such as age, height, weight, body mass index (BMI), and duration of illness followed the normal distribution $(\mathrm{P}>0.05)$. Using independent samples $\mathrm{t}$ test showed that 2 groups were matched regarding background characteristics at the baseline (Table 1).

Independent samples t test was also applied to check the differences between the groups regarding pain intensity and the Oswestry disability index at the baseline. Results showed no significant difference between 2 groups regarding these 2 outcome measures at the beginning of the interventions (Table 2).

To compare the group differences regarding the measures of the study variables at the beginning and the end of treatment, independent samples $t$ test was used.

Table 1. Comparison of variables in the study groups.

\begin{tabular}{|c|c|c|c|}
\hline Variable & Group & Mean (SD) & $P$ value \\
\hline & Intervention 1 & $39.00(8.90)$ & \\
\hline \multirow[t]{3}{*}{ Age $(y)$} & & & 0.39 \\
\hline & Intervention 2 & $41.60(7.50)$ & \\
\hline & Intervention 1 & $76.07(15.43)$ & \\
\hline \multirow[t]{3}{*}{ Weight (kg) } & & & 0.49 \\
\hline & Intervention 2 & 71.93(16.94) & \\
\hline & Intervention 1 & $168.80(14.24)$ & \\
\hline \multirow[t]{2}{*}{ Height (cm) } & & & 0.19 \\
\hline & Intervention 2 & $170.13(6.46)$ & \\
\hline \multirow{3}{*}{ Body mass index $\left(\mathrm{kg} / \mathrm{m}^{2}\right)$} & Intervention 1 & $26.92(5.46)$ & \\
\hline & & & 0.31 \\
\hline & Intervention 2 & $24.80(4.53)$ & \\
\hline \multirow{3}{*}{ The illness duration (mon) } & Intervention 1 & $20.07(11.12)$ & \\
\hline & & & 0.90 \\
\hline & Intervention 2 & 19.60(9.97) & \\
\hline
\end{tabular}


Table 2. Distribution of response variables in the study groups.

\begin{tabular}{cccc}
\hline Variable & Group & Mean (SD) & P value \\
\hline Pain & Intervention 1 & $7.46(1.68)$ & 0.53 \\
& Intervention 2 & $7.81(1.83)$ & \\
Oswestry disability & Intervention 1 & $50(0.08)$ & 0.92 \\
& Intervention 2 & $51(0.18)$ & \\
\hline
\end{tabular}

Assessment of differences regarding the severity of pain after the intervention, between the groups

Both groups showed a significant decrease in the pain severity. As shown in Figure 1, the average intensity of pain in the control group was 7.81 at the beginning of the treatment, and then, dropped to $2.64(\mathrm{P}=0.03)$. However, in the small ball exercise group, pain intensity was 7.46 at the beginning of treatment, then, decreased to $1.86(\mathrm{P}<0.001)$ at the end of treatment. Also a significant difference was observed between 2 intervention groups ( $\mathrm{P}=0.004)$. It means that both exercise programs in addition to electrotherapy, could reduce the pain severity, but more reduction was observed in the small ball exercise program.

Assessment of differences in the Oswestry disability index after the intervention, between the groups

Both groups showed a significant decrease in the Oswestry disability index. As shown in Figure 2, this index average for both groups was about $50 \%$ at the beginning of the treatment, then, reached $20 \%$ in the control group $(\mathrm{P}=0.01)$ and $11 \%$ in the small ball exercise group $(\mathrm{P}=0.02)$ at the end of treatment. Also, a significant difference was observed between 2 intervention groups $(\mathrm{P}=0.03)$. It means that both exercise programs in addition to electrotherapy, could reduce the Oswestry dis- ability index, but more reduction was observed in the small ball exercise program.

Reduction in pain and disability after the follow-up period persisted in both intervention groups, but more reduction was observed in the small ball exercise program $(\mathrm{P}<0.001)$.

Moreover, according to the patients' opinions, the exercises with small ball ranked higher considering its impact, ease of implementation, and desirability $(\mathrm{P}=0.01)$.

\section{Discussion}

Overall, it was determined that although both small ball and Williams exercise programs, along with routine electrotherapy, were effective in decreasing pain and disability in patients with CNSLBP, small ball exercises were more effective.

Numerous interventions are available to treat patients with chronic low back pain, but most of them have a modest effect on reducing pain and disability in patients with CNSLBP [20]. There are several drugs to relieve pain and improve function in patients with LBP, but due to the side effects caused by high doses and prolonged use of painkillers and other drugs commonly used, long-term

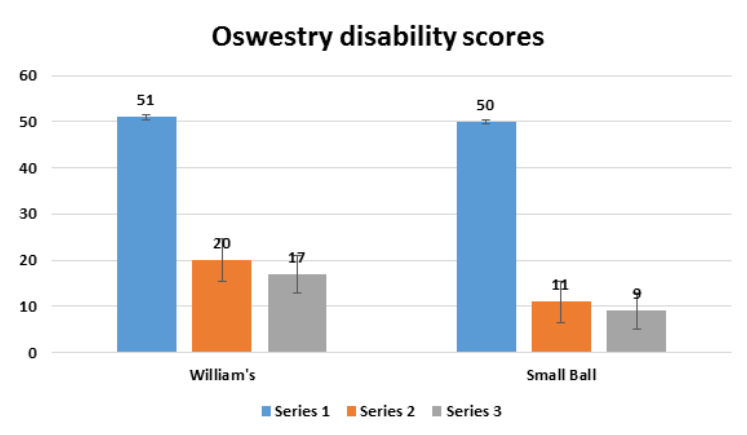

PHYSICAL TREA TMENTS

Figure 2. The mean scores of Oswestry disability measured in the Williams exercise group compared to small ball exercise group (15 patients in each group).
Figure 1. The means of pain severity measured in the Williams exercise group compared to small ball exercise group (15 patients in each group). 
use of the drugs is not reasonable and in such patients need non-pharmacological treatment strategies [21].

In various studies and different populations, it is found that applying rehabilitation programs for low back pain, and implementation of healthy lifestyle instructions are effective and more preferable than aggressive treatments, including spine operations [2, 3, 20, 21]. Exercise programs through activating and balancing muscle forces and stabilizing the spine can help resolve dysfunctions and improve the patients`symptoms and complaints [7, $11,13]$. Physiotherapy interventions, including exercise therapy in patients with LBP, are effective to relieve pain and improve functions [21-23].

In line with the results of the current study, there is plenty of evidence to suggest the active rehabilitation approaches to patients with chronic low back pain [20-22]. However, exercise programs to treat chronic low back pain in terms of duration, frequency, severity, and type are different; generally, exercise therapy is effective in reducing pain and improving functional abilities in such patients [24-26]. Studies also show the effects of exercise on postural control, electrical activity of muscles, improving neuromuscular control, and motor control [13, 27, 28]. Some types of specific exercises, which activate stabilizer muscles around the spine, are effective and also preferable compared to general routine exercises that patients with chronic non-specific low back pain do [29, 30]. A metaanalysis in 2012 confirmed the preference of core stability exercise versus general exercise for chronic LBP [31].

A clinical trial compared the effects of 3 types of treatment, including general exercises, stability exercises, and spinal manipulation therapy on 223 patients aged 18 to 80 years with chronic low back pain. Each patient was treated in 12 sessions for 8 weeks. Assessment tools included VAS, RMDQ, and PSFS. Immediately after 8 weeks, stabilization exercises group and manual therapy group had better outcomes than the public exercise group, but after 6 and 12 months follow-up, there was no significant difference between the 3 groups [32].

Although the current study confirmed the effect of physiotherapy and exercise on pain and dysfunction in LBP, more research is needed to consider the long-term effects and also other outcome measures. Van Middelkoop et al. conducted a study to give an overview on the effectiveness of exercise therapy in patients with low back pain. They showed that exercise therapy affected pain reduction and functional improvement in patients with chronic low back pain, although the effects were small and remained unclear which subgroups of patients benefited the most from a specific type of treatment They found no evidence that one particular type of exercise therapy was clearly more effective than others [32].

Chou et al. performed a review on noninvasive treatments for LBP from 2008 to 2015; they concluded that noninvasive treatments for LBP were associated with small to moderate, and primarily short-term effects on pain versus placebo, sham, wait list, or no treatment. Effects on function were generally smaller than effects on the pain. They suggested more research to understand optimal selection of treatments, effective combinations and sequencing of treatments, and effectiveness on outcomes other than pain and function [22].

Review of the exercise programs in the literature showed that some were neither specific nor detailed training. For example, some exercises were active public exercises for abdominal and lumbar muscles in standing, sitting, prone, supine positions, etc. Some were designed for a total of 3 months and mostly, patients were asked to do exercises at home, or only 4 to 6 sessions of treatment were under supervision in the physiotherapy clinic. Small ball exercise program recruits different muscle activations, and is easy to get trained and performed by patients. The current study added evidence that exercise therapy is an effective way to manage musculoskeletal dysfunctions, including low back pain [7, 11, 13, 19, 25].

Imbalance of lumbar and pelvic muscles causes dysfunctions in these areas. Most of the muscles in these regions act on the lower extremities as well. The current study introduced a new therapeutic exercise program that, according to the results, had greater influence on patients' signs and symptoms. The involved mechanism should activate and strengthen the weak muscles, relax tight muscles, and correct muscle activation patterns around the low back and pelvis. Small ball exercise program, by recruiting a good set of muscles, balances the muscle forces inserted to back and pelvic regions and results in further improvement of patients. Small ball exercise program, compared to the Williams exercise, obtained a higher rank, considering its effect on patients' improvement and also the patients' opinion regarding its impact, ease of implementation, and desirability. In this regard, the exercise program can be arbitrated for innovation and contribution to better understanding of the cumulative effect of muscle forces on lumbopelvic region.

There are few reliable studies on comparing therapeutic exercise programs in musculoskeletal disorders; previous reviews highlighted the necessity of good quality studies to provide a valid theory about a certain superiority of one exercise therapy to recover bothersome symp- 
toms in patients with nonspecific chronic low back pain $[22,31,32]$. According to this need assessment, the current study was conducted as a clinical trial with a control group, controlled conditions, and random allocation of patients into the intervention groups.

The responsible physiotherapist was not involved in assessments, and also the research fellow and patients in the current study were not aware of the type of treatment (groupings); thus, the study was conducted double-blind. The validity and reliability of the instruments of VAS and the Oswestry disability index for patients with low back pain had been already determined [14-16]. Both assessment instruments were based on patient's report, which can be considered as a limitation for the current study. It is suggested that future studies use electromyography to show muscle activity pattern, ultrasonography to measure the muscle size and also evaluating biomechanical characteristics, and objective clinical assessment procedures to assess patients' improvement.

Combining other interventions with physiotherapy and exercise therapy may increase the efficacy; a study showed that spinal manipulation provided better short- and longterm functional improvement, and more pain relief in the follow-up than either back school or individual physiotherapy. It can be considered as a suggestion for future studies.

The current study proved beneficial effects of the Williams exercise with routine physiotherapy in patients with nonspecific chronic low back pain, and also showed that substituting these exercises with small ball exercises could enhance the effectiveness of treatment influence on pain and disability. Small ball exercise program could be also shown better in the follow up, and it was ranked higher regarding its impact, ease of implementation and desirability by the patients.

\section{Clinical implication}

The current study compared the effects of 2 exercise therapy programs along with physical therapy on patients with CNSLBP and confirmed the efficacy of the 2 exercise programs. However, due to higher influence of small ball exercises than the Williams exercises, the current study offered a more effective program to patients and the medical society, especially the physiotherapists. In addition, the exercises with small ball provide faster healing process.

\section{Acknowledgements}

The authors thank all participants for their collaboration. The study was supported by the Department of Sports
Biomechanics, Faculty of Physical Education and Sports Sciences, the Islamic Azad University, Central Tehran Branch, Tehran, Iran. Authors also thank the colleagues who cooperated to conduct the research in clinics.

\section{Conflict of Interest}

The authors declared no Conflict of interests.

\section{References}

[1] Hoy D, March L, Brooks P, Blyth F, Woolf A, Bain C, et al The global burden of low back pain: Estimates from the Global Burden of Disease 2010 study. Annals of the Rheumatic Diseases. BMJ. 2014; 24; 73(6):968-74. doi: 10.1136/annrheumdis-2013-204428

[2] Ibrahim T, Tleyjeh IM, Gabbar O. Surgical versus nonsurgical treatment of chronic low back pain: A meta-analysis of randomised trials. International Orthopaedics. 2006; 32(1):107-13. doi: 10.1007/s00264-006-0269-6

[3] Van Tulder MW, Koes BW, Bouter LM. Conservative treatment of acute and chronic nonspecific low back pain. Spine. 1997; 22(18):2128-56. doi: 10.1097/00007632-19970915000012

[4] Volinn E1, Turczyn KM, Loeser JD. Theories of back pain and health care utilization. Neurosurgery clinics of North America. 1991; 2(4):739-48.

[5] Radebold A, Cholewicki J, Polzhofer GK, Greene HS. Impaired postural control of the lumbar spine is associated with delayed muscle response times in patients with chronic idiopathic low back pain. Spine. 2001; 26(7):724-30. doi: 10.1097/00007632-200104010-00004

[6] Chien JJ, Bajwa ZH. What is mechanical back pain and how best to treat it? Current Pain and Headache Reports . 2008, 12(6):406-11. doi: 10.1007/s11916-008-0069-3

[7] Panjabi MM. The stabilizing system of the spine. Part I. Function, dysfunction, adaptation, and enhancement. Journal of Spinal Disorders. 1992; 5(4):383-9. doi: 10.1097/00002517199212000-00001

[8] Nourbakhsh MR, Arab AM. Relationship between mechanical factors and incidence of low back pain. Journal of Orthopaedic \& Sports Physical Therapy. 2002; 32(9):447-60. doi: 10.2519/jospt.2002.32.9.447

[9] Triano JJ, Schultz AB. Correlationof objective measure of trunk motion and muscle function with low-back disability ratings. Spine. 1987; 12(6):561-5. doi: 10.1097/00007632198707000-00010

[10] O'Sullivan P. Diagnosis and classification of chronic low back pain disorders: Maladaptive movement and motor control impairments as underlying mechanism. Manual Therapy. 2005; 10(4):242-55. doi: 10.1016/j.math.2005.07.001

[11] Comerford MJ, Mottram SL. Functional stability re-training: principles and strategies for managing mechanical 
dysfunction. Manual Therapy. 2001; 6(1):3-14. doi: 10.1054/ math.2000.0389

[12] Hodges PW, Richardson CA. Inefficient muscular stabilization of the lumbar spine associated with low back pain. Spine. 1996; 21(22):2640-50. doi: 10.1097/00007632199611150-00014

[13] Richardson CA, Jull GA. Muscle control-pain control. What exercises would you prescribe? Manual Therapy 1995; 1(1):2-10. doi: 10.1054/math.1995.0243

[14] Price DD, McGrath PA, Rafii A, Buckingham B. The validation of visual analogue scales as ratio scale measures for chronic and experimental pain. Pain. 1983; 17(1):45-56. doi: 10.1016/0304-3959(83)90126-4

[15] Fairbank JC, Couper J, Davies JB, O'Brien JP. The oswestry low back pain disability questionnaire. Physiotherapy. 1980 66(8):271-3.

[16] Mousavi SJ, Parnianpour M, Mehdian H, Montazeri A, Mobini B. The oswestry disability index, the Roland-Morris disability questionnaire, and the Quebec back pain disability scale: translation and validation studies of the Iranian versions. Spine. 2006; 31(14):454-459. doi: 10.1097/01. brs.0000222141.61424.f7

[17] Foster NE, Thompson KA, Baxter GD, Allen JM. Management of nonspecific low back pain by physiotherapists in Britain and Ireland. Spine. 1999; 24(13):1332. doi: 10.1097/00007632-199907010-00011

[18] Robertson V, Ward A, Low J, Reed A. Electrotherapy explained, principles and practice. $4^{\text {th }}$ ed. Edinburgh: Elsevier Butterworth-Heinemann, 2006.

[19] Steiger F, Wirth B, de Bruin ED, Mannion AF. Is a positive clinical outcome after exercise therapy for chronic non-specific low back pain contingent upon a corresponding improvement in the targeted aspect(s) of performance? A systematic review. European Spine Journal. 2011; 21(4):575-98. doi: 10.1007/s00586-011-2045-6

[20] Van Middelkoop M, Rubinstein SM, Kuijpers T, Verhagen AP, Ostelo R, Koes BW, et al. A systematic review on the effectiveness of physical and rehabilitation interventions for chronic non-specific low back pain. European Spine Journal. 2010; 20(1):19-39. doi: 10.1007/s00586-010-1518-3

[21] Chou R. Diagnosis and Treatment of Low Back Pain: A Joint Clinical Practice Guideline from the American College of Physicians and the American Pain Society. Annals of Internal Medicine. American College of Physicians; 2007; 147(7):478. doi: 10.7326/0003-4819-147-7-200710020-00006

[22] Chou R, Deyo R, Friedly J, Skelly A, Hashimoto R, Weimer $M$, et al. Nonpharmacologic therapies for low back pain: a systematic review for an american college of physicians clinical practice guideline. Annals of Internal Medicin. American College of Physicians. 2017. doi: 10.7326/m16-2459

[23] Torstensen TA, Ljunggren AE, Meen HD, Odland E, Mowinckel P, af Geijerstam S. Efficiency and Costs of Medical Exercise Therapy, Conventional Physiotherapy, and Self-Exercise in Patients With Chronic Low Back Pain. Spine. 1998 23(23):2616-24. doi: 10.1097/00007632-199812010-00017

[24] Barr KP, Griggs M, Cadby T. Lumbar Stabilization. American Journal of Physical Medicine \& Rehabilitation. 2007; 86(1):72-80. doi: 10.1097/01.phm.0000250566.44629.a0.
[25] Koumantakis GA, Watson PJ, Oldham JA. Trunk muscle stabilization training plus general exercise versus general exercise only: Randomized controlled trial of patients with recurrent low back pain. Physical Therapy. 2005; doi: $10.1093 / \mathrm{ptj} / 85.3 .209$

[26] Stevens VK, Coorevits PL, Bouche KG, Mahieu NN, Vanderstraeten GG, Danneels LA. The influence of specific training on trunk muscle recruitment patterns in healthy subjects during stabilization exercises. Manual Therapy. 2007; 12(3):271-9. doi: 10.1016/j.math.2006.07.009

[27] Hall L, Tsao H, MacDonald D, Coppieters M, Hodges PW. Immediate effects of co-contraction training on motor control of the trunk muscles in people with recurrent low back pain. Journal of Electromyography and Kinesiology. 2009; 19(5):763-73. doi: 10.1016/j.jelekin.2007.09.008

[28] Karimi N, Talimkhani A, Mosallanezhad Z, Arab AM. The effects of consecutive supervised functional lumbar stabilizing exercises on the postural balance and functional disability in low back pain. Iranian Rehabilitation Journal. 2014 12(20): $21-27$

[29] Nabavi N, Mosallanezhad Z, Mohseni Bandpei M, Rahgozar M, Jaberzadeh Sh. The effect of two different exercise programs on pain intensity and muscle dimensions in patients with chronic low back pain: A randomized controlled trial. Journal of Manipulative and Physiological Therapeutics (In Press).

[30] Wang X-Q, Zheng J-J, Yu Z-W, Bi X, Lou S-J, Liu J, et al. A meta-analysis of core stability exercise versus general exercise for chronic low back pain. Public Library of Science (PLoS); 2012; 7(12):52082. doi: 10.1371/journal.pone.0052082

[31] Van Middelkoop M, Rubinstein SM, Verhagen AP, Ostelo RW, Koes BW, van Tulder MW. Exercise therapy for chronic nonspecific low-back pain. Best Practice \& Research Clinical Rheumatology. 2010; 24(2):193-204. doi: 10.1016/j. berh.2010.01.002

[32] Cecchi F, Molino-Lova R, Chiti M, Pasquini G, Paperini A, Conti AA, et al. Spinal manipulation compared with back school and with individually delivered physiotherapy for the treatment of chronic low back pain: a randomized trial with one-year follow-up. Clinical Rehabilitation.ations; 2010; 24(1):26-36. doi: 10.1177/0269215509342328 